\title{
DEBATE SOBRE LA INCLUSIÓN \\ DEL COOPERATIVISMO DENTRO DE LAS POLIITICAS DE LA NUEVA RURALIDAD EN AMÉRICA LATINA
}

Francisco de Borja García-García

Universidad de Cádiz

España 
Panorama Económico, Vol. 25 - No. 3 (Julio - Septiembre de 2017), pp. 357-380

Francisco de Borja García-García

\title{
Debate sobre la inclusión del cooperativismo dentro de las políticas de la Nueva Ruralidad en América Latina
}

\begin{abstract}
Resumen
La sociedad rural en América Latina ha estado enfrentada a graves problemas, como la pobreza ru-ral, los cambios productivos agrícolas, el éxodo al mundo urbano o la entrada de los pequeños y medianos agricultores en las cadenas de valor. Para solventar dichos problemas surge la definición de Nueva Ruralidad que estudia las políticas que satisfacen las necesidades sociales del mundo rural. Por otro lado, la Economía Social, cuya figura jurídica más representativa es la cooperativa, propone que el interés social esté por encima de los intereses económicos y estudia como satisfacer las necesidades de las personas en riesgo de exclusión social. En este artículo, en primer lugar se realiza un estudio comparativo y crítico de cómo se han desarrollado las políticas rurales en América Latina. En segundo lugar se estudia como las cooperativas agrarias influyen en la producción agrícola en algunos países de América Latina. Por último, se proponen una serie de medidas que pueden aplicarse para que los modelos cooperativos participen en América Latina Palabras clave: Economía social, cooperativas, Nueva Ruralidad, América Latina, Políticas públicas.

Clasificación JEL: A13, R11, O18, P25
\end{abstract}

\section{Débat sur l'inclusion du coopérativisme dans les politiques de la nouvelle ruralité en Amérique latine}

\begin{abstract}
Rural society in Latin America has been confronted with serious problems, such as rural poverty, agricultural production changes, the exodus to the urban areas or the inclusion of small and mediumsized farmers in the value chains. To solve these problems comes the definition of New Rurality which studies the policies that satisfy the social needs of the rural world. On the other hand, the Social Economy, whose most representative legal figure is the cooperative, proposes that social interest is above economic interests and studies how to meet the needs of people at risk of social exclusion. First, it has been made a comparative and critical study of how rural policies in Latin America have been developed. Second, we study how agricultural cooperatives influence agricultural production in some Latin American countries. Finally, some measures are proposed to foster the application of cooperative models within Latin America Keywords: Social economy, cooperatives, New Rurality, Latin America, Public policies.

JEL Classification: A13, R11, O18, P25
\end{abstract}

\section{L'économie sociale dans la décentralisation des politiques sociales: l'expérience des États- providence européens}

\begin{abstract}
Résumé
La société rurale en Amérique latine a été confrontée à de graves problèmes, tels que la pauvreté rurale, les changements dans l'agriculture de production, l'exode de la population rurale vers le monde urbain ou l'entrée des petits et moyens agriculteurs dans les chaînes de valeur. Pour résoudre ces problèmes, la définition intitulée Nouvelle ruralité apparaît, qui étudie les politiques qui répondent aux besoins sociaux du monde rural. D'autre part, l'économie sociale, dont le statut juridique le plus représentatif est la coopérative, propose que l'intérêt social soit supérieur aux intérêts économiques et étudie les moyens de répondre aux besoins des personnes menacées d'exclusion sociale. Dans cet article, nous examinons d'abord une étude comparative et critique de la façon dont les politiques rurales en Amérique latine ont été élaborées. Deuxièmement, nous étudions comment les coopératives agricoles influencent la production agricole de certains pays d'Amérique latine. Enfin, une série de mesures pouvant être appliquées pour que des modèles coopératifs participent aux mesures en Amérique latine sont proposées.

Mots-clés: Économie sociale, coopératives, nouvelle ruralité, Amérique Latine, politiques publiques.
\end{abstract}

Nomenclature JEL: A13, R11, O18, P25 


\section{Debate sobre la inclusión del cooperativismo dentro de las políticas de la Nueva Ruralidad en América Latina}

INFORMACIÓN DEL ARTíCULO

Recepción de artículo: 10/04/2017

Concepto de evaluación: 25/05/2017

Aceptación de artículo: 25/06/2017
Francisco de Borja García-García

Universidad de Cádiz

España

\section{INTRODUCCIÓN}

Las dificultades sociales del mundo rural en América Latina se componen por una serie de problemas estructurales como la exclusión social, el empeoramiento de las condiciones de vida de sus ciudadanos, la distribución equitativa de las tierras o la falta de valor añadido de recursos productores y naturales. Como consecuencia, existe una baja calidad de vida traducida en altos niveles de pobreza rural. Estas circunstancias han conducido a las poblaciones rurales hacia el envejecimiento de la población autóctona así como al abandono de actividades agrícolas como motores del desarrollo social del territorio (Informe Organización de las Naciones Unidas para la Alimentación y la Agricultura, 2014) ${ }^{1}$.

El estudio a modo de diagnóstico realizado por la FAO con carácter especial en 2014, profundiza sobre estos problemas enfocando posibles soluciones en el largo plazo. No obstante, a los problemas que ya menciona la FAO, se le añaden los datos oficiales del informe de la Oficina de Estudios y Políticas Agrarias (ODEPA 2013)², sumándose a los ya existentes problemas sociales del mundo rural Latino Americano los cambios climáticos, productivos y laborales que afectan al mundo agrícola a nivel mundial.

\footnotetext{
* Autor para correspondencia

Correos electrónicos: franciscoborja.garciagarcia@alum.uca.es

1 Informe de la Organización de las Naciones Unidas para la Alimentación y la Agricultura titulado "Pobreza Rural y políticas Públicas en América Latina y el Caribe" publicado en 2014.

2 Estudio encargado por la Oficina de Estudios y Políticas Agrarias del Ministerio Chileno titulado "Cambio climático Impacto en la Agricultura Helada y Sequía".
} 
Ante este escenario social, y en un contexto de agricultura globalizado, aparece la exigencia de políticas conjuntas entre los organismos internacionales Latinoamericanos para solucionar los problemas rurales. La necesidad de nuevas propuestas crea un debate sobre cuáles son las pautas de actuación más convenientes para solucionar la complicada situación existente dentro del mundo rural en Latino América.

En este punto se centran dos campos de investigación en América Latina que buscan paliar los problemas emergentes dentro del mundo rural. Por un lado, el concepto de Nueva Ruralidad, NR, surge como un posible paquete de medidas públicas que procuran satisfacer las demandas sociales en el mundo rural. Y por otro, el concepto de la Economía Social o Solidaria, no tan centrada en la agricultura y cuya máxima expresión se centra en el cooperativismo busca poner el desarrollo social por encima de los intereses económicos además de proteger a las personas en riesgo de exclusión social (Chaves, 2008).

Por lo tanto y en este marco que exige soluciones en el corto plazo a un problema prolongado en el tiempo, se corre el riesgo de queambos políticas, a pesar de tener objetivos en común, no interaccionen entre sí.

El tema objeto de este estudio es de la más absoluta actualidad y puede significar una respuesta a uno de los problemas presentes en la sociedad y economía rural. Por tanto, este artículo pretende tener una aportación real y práctica al ámbito de la Economía Social y al desarrollo territorial en Latino América.

\section{METODOLOGÍA}

El objetivo de este artículo es proponer a la Economía Social como una de las medidas que mejore las condiciones sociales dentro de la Nueva Ruralidad.

Para ello, en primer lugar. se ha analizado las aportaciones de los principales autores, a partir de la revisión bibliográfica, consultando bases de datos especializadas tales como ISI WEB of Knowledge, SCOPUS y Dialnet, entre otros, definiendo qué es la Nueva Ruralidad y la Economía Social.

A partir de estas definiciones se realizan dos bloques que interpretan el impacto social cosechado de ambas definiciones en los últimos años en América Latina.

Para conocer cómo se han desarrollado las medidas de la Nueva Ruralidad, se han tomado ejemplos de políticas rurales desde dos enfoques:

- En primer lugar, se estudian los trabajos internacionales tanto de la FAO como de la Comisión Económica Para América Latina y el Caribe, las conclusiones sacadas y las propuestas llevadas a cabo. De los mismos se analizan sus objetivos, sus conclusiones y se toman datos para un posterior análisis cuantitativo.

- En segundo lugar, se analizan casos de políticas diversas a nivel nacional. A partir de la interpretación de las diversas políticas, se realiza un análisis cuantitativo con algunas variables que explican la pobreza rural comprendidas en dos periodos; primer y segundo quinquenio del siglo XXI. A partir de los mismos se realiza un análisis Cluster utilizando el método "Vecino más cercano" para clasificar los distintos países en tres grupos.

A partir de los grupos obtenidos por los Clusters, se toma al menos uno de los países de los grupos, para estudiar el impacto de la economía social dentro del mundo rural. 
- En primer lugar, se observan los datos generales de la Economía Social en América latina, y se realiza una tabla comparativa de algunas variables que explican el impacto del cooperativismo agrario en los países estudiado.

- En segundo lugar, y a partir de la revisión literaria de diagnósticos tanto de organismos internacionales como la Organización Internacional del Trabajo, o CEPAL, Aliance Cooperative International Américas o CICOPA como organismos nacionales INFOCOOP, se realiza un análisis de la relación existente entre el cooperativismo agrario y la pobreza rural.

Por último, a partir de los resultados obtenidos se procede a proponer una serie de recomendaciones para introducir el cooperativismo dentro de las políticas de la Nueva Ruralidad.

\section{DEFINICIÓN DE LA NUEVA RURALI- DAD EN LATINO AMÉRICA}

La sociedad rural ha estado comprendida históricamente por aquellos territorios habitables y administrables cuya tradición no puede ser considerada como urbana (Bonnal, 2003). Sin embargo varios autores defienden (Bretón, 2005), (Gramont, 2004), (Delgado,1999) queen el mundoglobalizado ha quedado anticuado este concepto de división entre sociedad urbana o rural. La tendencia actual dentro del desarrollo territorial habla sobre una única sociedad globalizada que tiene necesidades heterogéneas, independientemente de cómo sea su entorno, apareciendo un nuevo debate teórico sobre cómo debe de ser la evolución del desarrollo territorial en las antiguas sociedades rurales para equipararse a lo que ofrece las sociedades urbanas (Kay, 2005).
El mundo de lo rural siempre ha estado muy ligado a la producción agropecuaria. La agricultura durante las décadas de los 80 y 90 sufre importantes cambios dentro de su modelo productivo fruto de la globalización (Ramírez-Miranda, 2014), (Pérez Correa, 2004) (Rivera, 2008). Aparece una nueva producción industrial agrícola enfocada a un mercadoglobalizado que exige un modelo latifundista y que se respaldada en el trabajo tecnológico y biológico optimizando la productividad y permitiendo su mercalización tanto en mercados nacionales como internacionales (Ballesteros, 2014).

Estos cambios de modelos productivos surgidos en Europa y Estados Unidos no tardarán en extenderse al resto del mundo. La necesidad de competir en un mercado agrícola que obedezca a leyes internacionales que protejan al consumidor han derivado la producción hacia un mercado más competitivo y estandarizado. También se ha transformado la urbanización del mundo rural, derivando en actividades que no son solo agrícolas e intentando satisfacer necesidades locales que antiguamente solo podían ser satisfechas en la ciudad (Gómez, 2001).

Sin embargo, en el entorno rural de América Latina, a la obligación de modernizar la producción agrícola y la necesidad de urbanizar los territorios rurales, se le suman antiguas problematicas locales como los conflictos territoriales armados en Colombia (Vargas, 2003) (Peña y Herrera, 2016), la falta de estabilidad política de Venezuela o Argentina (Rock, 1999), y la ubicación de los pueblos originarios en Chile o Perú (Fontenoy, 2012), (Noa, 2015). A esta prolongada situación de inestabilidad se le añade la falta de interés de políticas públicas rurales en detrimento por los planes de industrialización. Esta falta de iniciativas estatales 
rurales entre la década de los setenta hasta los noventa, dejó un vacío institucional agrario que luchase efectivamente por satisfacer las necesidades sociales rurales de Latinoamérica (Araoz, 2002).

Las consecuencias de esta falta de políticas rurales se traducen en una sociedad con altos niveles de pobreza rural (tabla 1):

Tabla 1: Evolución de población rural bajo la línea de pobreza en países de América Latina

\begin{tabular}{|l|c|c|c|c|}
\hline \multirow{2}{*}{ País } & \multicolumn{2}{c|}{$\begin{array}{c}\text { Porcentaje de población rural bajo la } \\
\text { línea de pobreza }\end{array}$} & $\begin{array}{c}\text { Porcentaje de la población rural bajo la } \\
\text { línea de la indigencia }\end{array}$ \\
\cline { 2 - 5 } & 2000 & 2010 & 2000 & 2010 \\
\hline Honduras & 86 & 79 & 68 & 62 \\
\hline Bolivia & 81 & 76 & 65 & 59 \\
\hline Nicaragua & 77 & 72 & 58 & 46 \\
\hline Paraguay & 74 & 67 & 53 & 47 \\
\hline Guatemala & 69 & 67 & 42 & 42 \\
\hline Colombia & 70 & 64 & 35 & 29 \\
\hline Perú & 70 & 60 & 37 & 28 \\
\hline El Salvador & 58 & 63 & 34 & 25 \\
\hline América Latina & 63 & 53 & 38 & 30 \\
\hline Ecuador & 59 & 46 & 40 & 23 \\
\hline México & 59 & 45 & 31 & 20 \\
\hline Rep. Dominicana & 56 & 45 & - & 22 \\
\hline Panamá & 55 & 44 & 35 & 15 \\
\hline Brasil & 59 & 39 & 27 & 9 \\
\hline Costa & 22 & 20 & 10 & 4 \\
\hline Rica & 27 & 10 & 9 & \\
\hline Chile & & & & \\
\hline
\end{tabular}

Fuente: Elaboración propia a partir de datos CEPAL, 2010

Los países con menores índices de población en exclusión social, Chile, Brasil y Costa Rica, no diferencian sus focos de pobreza entre territorios urbanos y rurales. Sin embargo, los países de menor desarrollo social concentran su pobreza dentro del mundo rural, Salvador, Nicaragua y Honduras (Tabla 2):

Tabla 2: Población bajo la línea de pobreza (LP), 1,5 veces LP y 2 veces LP (\% población total)

\begin{tabular}{|l|c|c|c|}
\hline \multirow{2}{*}{\multicolumn{1}{c|}{ País }} & LP & Población por debajo de \\
\cline { 2 - 4 } & 11,5 & 25,9 & 2 LP \\
\hline Chile (2009) & 16,4 & 33.0 & 40,2 \\
\hline Costa Rica (2008) & 25,8 & 40,3 & 46,9 \\
\hline Brasil (2008) & 34,8 & 55,8 & 51,8 \\
\hline México (2008) & 47,9 & 69,8 & 69,2 \\
\hline El Salvador (2009) & 61,9 & 78,8 & 81,4 \\
\hline Nicaragua (2005) & 68,9 & 81,4 & 86,4 \\
\hline Honduras (2007) & & 88,4 \\
\hline
\end{tabular}

Fuente: FAO.ARLC con base encuesta hogares. 
En la actualidad, se estudia el desarrollo territorial rural en Latinoamérica como un conjunto de paquete de medidas que intentan promover el desarrollo sostenible en el mundo del campo. Varios autores (Matás, 2013), (Mejía, 2016), (MaríVidal, 2013), (Quijano, 2014) sostienen que el mejoramiento de las condiciones sociales de los espacios rurales pasa por modernizar la producción agrícola, así como traer nuevos servicios y mercados sobre los territorios urbanos.

No obstante, y como describe Rivera 2012, las necesidades sociales rurales no pueden ser satisfechas sin que surja una destrucción y transformación del mundo del campo. Las políticas a tomar tienen diversos objetivos dependiendo del enfoque. Por lo tanto, y para que exista este ansiado desarrollo sostenible del mundo urbano, hay que llevar a cabo una serie de iniciativas que erradiquen los siguientes problemas (Cuadro 1).

\section{El IMPACTO DE LA NUEVA RURALIDAD EN LATINO AMÉRICA}

La NR está comprendida por una serie de políticas miradas desde distintos enfoques cuyo fin es satisfacer los nuevos problemas del mundo rural. Estas se han enfocado en satisfacer demandas locales urgentes dentro de los territorios conflictivos con políticas cuyo objetivo principal son la erradicación de la pobreza, el rejuvenecimiento de la población o la involucración en el mercado de los pequeños y medianos agricultores.

Las medidas llevadas a cabo durante la década de los noventa, apostaron por la descentralización estatal llevando a cabo programas de desarrollo local (Molotla, 2013). Sin embargo, la inestabilidad política, sumada con los diferentes organismos nacionales e internacionales que han intervenido en las políticas públicas agrarias y rurales, dificultaron que estas medidas alcanzaran los objetivos esperados.

A continuación se estudian casos de medidas políticas que buscan solventar los problemas sociales rurales, tanto a nivel internacional, como nacional.

\section{A) Políticas Internacionales}

En Latino América existen una gran diversidad de organismos internacionales pertenecientes al desarrollo territorial que han diagnosticado el mundo rural. No obstante, a pesar del énfasis puesto por estos organismos, y ante la falta de considerables fondos públicos para financiar políticas directas, su trabajo

\section{Cuadro 1: Problemas a solventar por la Nueva Ruralidad}

\begin{tabular}{|c|c|c|}
\hline Social & Productivo & Natural \\
\hline $\begin{array}{l}\text { - Crecimiento de la brecha } \\
\text { entre ricos y pobres. } \\
\text { - Exclusión social entre los } \\
\text { pequeños y medianos } \\
\text { agricultores. } \\
\text { - Concentración de la pobreza } \\
\text { en el mundo rural. } \\
\text { - Migración masiva del mundo } \\
\text { rural }\end{array}$ & $\begin{array}{l}\text { - Falta de adaptación en la } \\
\text { transformación de modelos } \\
\text { productivos agrícolas. } \\
\text { - Necesidad de competir en un } \\
\text { mercado internacional. } \\
\text { - Necesidad de tecnologías } \\
\text { biológicas. }\end{array}$ & $\begin{array}{l}\text { - Falta de ecologismo popular. } \\
\text { - Crisis ambiental. }\end{array}$ \\
\hline
\end{tabular}

Fuente: Elaboración propia. 
se ha centrado en el diagnóstico y la recomendación.

La FAO en 2009 hace un diagnóstico titulado "Boom Agrícola y Persistencia de la Pobreza Rural". En el mismo se critica la concentración de la tierra y la productividad agrícola, diagnosticando que el modelo de globalización agrícola había conducido a los sistemas productivos agrarios al latifundio de grandes corporaciones que se apropian de toda la cadena de valor, dificultando la entrada al mercado entre los pequeños y medianos agricultores.

"Políticas de Mercado de Trabajo y Pobreza Rural en América Latina" (FAO. CEPAL. OIT, 2010 y 2012) diagnostica que los motivos de los altos índices pobreza rural se debe a las bajas condiciones laborales. Para llegar a esta deducción se estudia el conjunto de las instituciones agrarias que participan en la elaboración de las políticas, y se toman en común una serie de variables como el salario mínimo agrario, el porcentaje de trabajo infantil o la discriminación femenina.

"El estado mundial de la agricultura y la alimentación"'(FAO, 2012) Un tercer estudio en esta línea, abordó las dinámicas del mercado de la tierra en América Latina y el Caribe, con base en 17 estudios por países mostrando que los niveles de concentración -y de extranjerización- de la tenencia de la tierray delosrecursosengeneralen la región, demostrando que ha aumentado en forma notable durante las dos últimas décadas, aunque con importantes variaciones subregionales, expresión también de los fenómenos que ocurre en las cadenas de valor, abriendo una interrogantes sobre el futuro de la agricultura campesina y la producción de alimentos básicos.
En los estudios de la FAO, 2014," Pobreza Rural y Políticas Públicas en América Latina y el Caribe" aprovecha el año de la Agricultura Familiar para incidir dentro de los problemas que más preocupan a la población rural. Por ello realiza una serie de encuestas a familias rurales de 12 países en América Latina, profundizando sobre cuáles son sus inquietudes, y según su opinión, como podría mejorar su forma de vida. Se concluye con que existe una población desencantada de lo rural y lo agrícola, con una gran tendencia a las migraciones urbanas y un alto envejecimiento poblacional.

"La Nueva ruralidad para Europa y su interés para América Latina": Tanto en la FAO 2014 como la CEPAL 2012, recomiendan la aplicación Leader para adjudicar fondos estatales. Sus objetivos son cercanos al modelo europeo, pero aplicándolo a la realidad rural de Latino América. A diferencia de Europa, no se prima tanto los objetivos de desarrollo natural sostenible, y se centra en la erradicación de la pobreza, el asentamiento de la población rural, y la necesidad de fomentar políticas de integración para los pueblos indígenas.

\section{B) Políticas nacionales.}

Las políticas nacionales de los distintos países Latino Americanos a pesar de tener una serie de puntos en común como la lucha contra la pobreza o la innovación agrícola no han tenido una planificación internacional conjunta como puede ser el caso de la Unión Europea (Carnero, 2015) (Soto Baquero, 2012). Resultado de ello, las medidas de intervención en el mundo rural han sido muy variadas. En el cuadro 2. se observa algunas medidas que han sido tomadas como ejemplo para este artículo: 


\section{Cuadro 2: Ejemplos de políticas rurales nacionales en Latino Americana.}

\begin{tabular}{|c|c|}
\hline País & Políticas \\
\hline Argentina & $\begin{array}{l}\text { El Estado argentino, basado en las corrientes agrícolas del post-consenso de } \\
\text { Washington, reconstruye en } 2009 \text { el Ministerio de Agricultura, Ganadería y Pesca } \\
\text { para promover ella Agricultura familiar (agricultura; ganadería) con financiación } \\
\text { internacional y estatal para el desarrollo rural. El modelo de actuación consiste en } \\
\text { presentar concursos con objetivos ya predefinidos para que los diferentes actores } \\
\text { locales postulen a dichos proyectos (Latuada, 2014) }\end{array}$ \\
\hline Brazil & $\begin{array}{l}\text { El Programa Nacional de Fortalecimento da Agricultura Familiar (PRONAF) surge en } \\
1995 \text { e incorpora en } 1999 \text { al Manual de Crédito Rural. Dicho programa orienta la } \\
\text { política agraria agrupando a los agricultores por especialidades. Una vez se haya } \\
\text { demostrado un plan de trabajo que compromete a colaborar a distintos agricultores, } \\
\text { el Estado brasileño da facilidades de créditos para la financiación agrícola (Zaar, 2010). }\end{array}$ \\
\hline Bolivia & $\begin{array}{l}\text { Dentro de los planes llamados "Saber Vivir" en } 2006 \text { el gobierno del Estado } \\
\text { Plurinacional de Bolivia emprende un proceso de "Revolución Agraria Comunitaria" } \\
\text { para impulsar una política nacional agraria. Dicho año se entregó a las comunidades } \\
\text { indígenas 3,2 millones de hectáreas de tierras. }\end{array}$ \\
\hline Chile & $\begin{array}{l}\text { Los Fondos de Innovación Agraria FIA aparecen en } 2010 \text { a través del Ministerio } \\
\text { Agrícola. Se encarga de presentar proyectos agrícolas con unos objetivos ya } \\
\text { predefinidos, para que los distintos actores nacionales pueden postular a una única } \\
\text { adjudicación. Con ello se pretende fomentar la competitividad agrícola, forzando la } \\
\text { cooperación y optimizando los fondos estatales. }\end{array}$ \\
\hline Ecuador & $\begin{array}{l}\text { La Agenda para laTransformación Productiva } 2010 \text { - } 2013 \text { presentada por el Consejo } \\
\text { Sectorial de la Producción, define las bases para el desarrollo productivo del país } \\
\text { en los próximos } 4 \text { años. Sus objetivos están dirigidos a transformar el patrón de } \\
\text { especialización con alto valor agregado, mejorar la competitividad de la producción } \\
\text { reduciendo las brechas intersectoriales, democratizar el proceso de acumulación, } \\
\text { maximizar la inversión y la infraestructura, propender a la eficiencia energética y } \\
\text { transversalizar la equidad y la inclusión en todas las políticas aplicables. Para ello el } \\
\text { Estado dedica fondos para crear nuevas instituciones centrales que hagan cumplir } \\
\text { dichos propósitos. }\end{array}$ \\
\hline
\end{tabular}

Fuente: Elaboración propia

Estas políticas agrarias están inspiradas en los modelos Leader europeos dejando que sean los actores locales quienes gestionen los recursos estatales. Sin embargo se observan modelos dispares:

\section{- Los casos Argentino y Chileno} muestran semejanzas al apostar por políticas con objetivos predefinidos que deben alcanzarse sacando las distintas financiaciones a concurso público.

- EL Estado Brasileño favorece políticas de financiación de agrupaciones. No obstante, se critica una falta de seguimiento de los fondos públicos ya que la adjudicación de la ayuda financiera no va seguida con unos objetivos a cumplir.

- El caso Ecuatoriano apuesta porque las instrucciones estatales sean quienes lleven a cabo los planes de desarrollo territorial rural alejándose del modelo Leader.

- El Estado Boliviano apuesta por la entrega de tierras para la integración de los pueblos indígenas. Sin embargo, no estipula unos objetivos para conseguir un modelo de sostenibilidad. 


\section{C) Resultado de las políticas rurales.}

Los diferentes diagnósticos y políticas regionales dejan unos resultados ambiguos que se traducen en realidades rurales dispares. Sin embargo, sí se observa unos objetivos repetidos en la propuesta de las políticas como la integración social de la población rural, sofocando la pobreza, la innovación agrícola y la mejora de las condiciones laborales de los campesinos.

Las diferentes políticas planteadas en los distintos países de Latino América no siempre han llevado los resultados esperados. Para el análisis de estos resultados, se toman los datos del CEPALSTAT. A partir de los mismos se dividen los datos en dos periodos de tiempo: el primer y el segundo quinquenio del nuevo siglo. Las variables elegidas han sido las siguientes:
- \% De la Población ocupada agrícola respecto a la población ocupada total: Aplica cual es la cantidad de población ocupada en el mundo rural para conocer el peso agrícola de la población ocupada del país.

- \% Incidencia de la pobreza rural a la situación de actividad económica: Indica la situación laboral de las personas bajo la línea de la pobreza separando entre la población desocupada y la ocupada. Con ello se observa cual es el porcentaje de la población que sigue bajo el umbral de la pobreza a pesar de estar empleada.

- Coeficiente de Gini de las poblaciones rurales: Mide las desigualdades de renta entre la población rural.

- Ingresos de hogares rurales en situación de pobreza: Diferencia la entrada de ingresos en hogares rurales bajo la línea de la pobreza, separando entre ingresos laborales, e ingresos no laborales.

Tabla 3: Algunas variables que explican la pobreza rural en Latino América.

\begin{tabular}{|c|c|c|c|c|c|c|c|c|c|c|c|c|}
\hline \multirow{3}{*}{ PAIS } & \multicolumn{2}{|c|}{$\begin{array}{c}\text { \%Población ocupada } \\
\text { agrícola respecto a la } \\
\text { población ocupada } \\
\text { total }\end{array}$} & \multicolumn{4}{|c|}{$\begin{array}{c}\text { \%Incidencia de la pobreza } \\
\text { rural a la situación de } \\
\text { actividad } \\
\text { económica }\end{array}$} & \multicolumn{2}{|c|}{$\begin{array}{c}\text { Coeficiente de Gini } \\
\text { de las poblaciones } \\
\text { Rurales }\end{array}$} & \multicolumn{4}{|c|}{$\begin{array}{l}\text { Ingresos de hogares rurales } \\
\text { en situación de pobreza }\end{array}$} \\
\hline & \multirow{2}{*}{$\begin{array}{c}1^{\circ} \\
\text { Quinquenio }\end{array}$} & \multirow{2}{*}{$\begin{array}{c}2^{\circ} \\
\text { Quinquenio }\end{array}$} & \multicolumn{2}{|c|}{ Ocupados } & \multicolumn{2}{|c|}{ Desocupados } & \multirow{2}{*}{$\begin{array}{c}1^{\circ} \\
\text { Quinquenio }\end{array}$} & \multirow{2}{*}{$\begin{array}{c}2^{\circ} \\
\text { Quinquenio }\end{array}$} & \multicolumn{2}{|c|}{ laboral } & \multicolumn{2}{|c|}{ No laboral } \\
\hline & & & $1^{\circ} \mathrm{Q}$ & $2^{\circ} \mathrm{Q}$ & $1^{\circ} \mathrm{Q}$ & $2^{\circ} \mathrm{Q}$ & & & $1^{\circ} \mathrm{Q}$ & $2^{\circ} \mathrm{Q}$ & $1^{\circ} \mathrm{Q}$ & $2^{\circ} \mathrm{Q}$ \\
\hline Bolivia & 85,7 & 75,3 & 77 & 73 & 76 & 78 & 0,48 & 0,42 & 83,4 & 86,9 & 16,6 & 13.0 \\
\hline Brasil & 75,6 & 67,4 & 46 & 31 & 62 & 50 & 0,55 & 0,52 & 81,7 & 70,8 & 18,3 & 29,2 \\
\hline Chile & 64,8 & 52,2 & 16 & 4 & 41 & 30 & 0,5 & 0,47 & 45 & 32,6 & 55 & 67,4 \\
\hline Guatemala & 55,9 & 53,6 & 58 & 57 & 77 & 53 & 0,47 & 0,53 & 69,5 & 73,5 & 30,5 & 26,5 \\
\hline Honduras & 66,4 & 61,5 & 81 & 73 & 81 & 78 & 0,52 & 0,578 & 81,6 & 64,6 & 18,4 & 35,4 \\
\hline R. Dominicana & 41,7 & 33,9 & 40 & 28 & 59 & 53 & 0,46 & 0,5 & - & - & - & - \\
\hline México & 44,3 & 35 & 45 & 35 & 58 & 46 & 0,49 & 0,49 & - & - & - & - \\
\hline Perú & 78 & 72,6 & 74 & 55 & 69 & 59 & 0,43 & 0,4 & - & - & - & - \\
\hline Panamá & 54,2 & 48,3 & - & - & - & - & - & - & - & - & - & - \\
\hline Paraguay & 63,6 & 59,1 & 65 & 60 & 73 & 64 & 0,54 & 0,58 & 87,9 & 79,8 & 12,1 & 20,2 \\
\hline Uruguay & 70,1 & 68,8 & - & 3 & - & 13 & - & - & - & - & - & -- \\
\hline Colombia & 60,3 & 65,2 & 59 & 52 & 73 & 70 & 0,54 & 0,49 & - & - & - & - \\
\hline Nicaragua & 64,8 & 68,5 & 69 & 63 & 80 & 62 & - & - & - & - & - & - \\
\hline El Salvador & 47,9 & 49 & 52 & 47 & 71 & 70 & - & - & - & - & - & - \\
\hline Ecuador & 68,6 & 68 & 51 & 38 & 66 & 56 & 0,43 & 0,44 & 84,2 & 69,6 & 15,9 & 30,4 \\
\hline Costa Rica & 35,1 & 35,9 & 14 & 9 & 41 & 37 & 0,48 & 0,46 & 60 & 59,8 & 40 & 40,2 \\
\hline
\end{tabular}

Fuente: Elaboración propia a partir de los Datos CEPALSTAT 2010. 
En primer lugar, se observa que la población agrícola disminuye en todos los países (excepto, Costa Rica). Por lo tanto, existe una tendencia al éxodo del mundo urbano. Estos datos, sumados a los diagnósticos internacionales, muestra que existe una tendencia de migración rural hacia el mundo urbano. La población que se mantiene en el territorio rural es aquella más envejecida que no tiene aspiraciones a cambiar su medio de vida (FAO, 2012). Por lo tanto, uno de los problemas que debe de tomar la Nueva Ruralidad es crear un entorno atractivo que reduzca la salida de la población rural.

En, al menos, Bolivia, Guatemala, Perú, Paraguay, Colombia y Nicaragua, más del $50 \%$ de la población agrícola ocupada es pobre. Por lo tanto, los ingresos percibidos por la actividad agrícola son insuficientes para salir del umbral de la pobreza. En el resto de países existe un alto porcentaje de población ocupada que no tiene una renta lo suficientemente alta como para no tener tal consideración. Los países con una ratio menor al 10\% de población ocupada pobre ${ }^{3}$ son Costa Rica, Chile y Uruguay.

A pesar de que existe una reducción en los índices de pobreza, las desigualdades, como se observa al ver el índice de Gini ver, no han disminuido notoriamente dentro del mundo rural. La pobreza rural ha bajado a mayor velocidad que las desigualdades de renta. En Guatemala, México y Paraguay las desigualdades han aumentado, aunque se hayan reducido los índices de pobreza. Por lo tanto, a pesar de que se puede hablar de una mejora social en el corto plazo, también existe un mercado agrícola que propone una estructura de mercado desigual al largo plazo.

La alta cantidad de ingresos no laborales dentro de las familias en situación de

3 La CEPAL en el periodo comprendido entre 2000-05, considera a personas bajo la línea de la pobreza aquellas cuyos ingresos no llegan a dólar por día. En 2005 se determinó un nuevo umbral de 1,25 dólar por día. pobreza indica que existe una barrera entre los pequeños y medianos agricultores para su entrada al mercado agrícola . Por lo tanto, al considerarse como mercados competitivos agrícolas a aquellos que se encuentran dentro de una cadena de valor añadido (FAO, 2010), el aumento de población empleada no ha sido productivo. Los medianos y pequeños agricultores no pueden profesionalizar su actividad agrícola, y esa falta de especialización hace que exista una alta probabilidad de que la renta laboral no permita superar el umbral de la pobreza.

Para poder clasificar como ha evolucionado la realidad rural como consecuencia de las políticas practicadas por los diferentes países, se aplica un análisis clúster empleando el método de "vecino más cercano" (dendograma 1 y dendograma 2).

Esta técnica se utiliza para crear grupos entre los países que nos permite facilitar el estudio posterior de cómo de beneficioso ha sido el impacto de las cooperativas en los territorios estudiados. Para ello, se han tomado los datos en dos momentos distintos para ver la evolución cronológica de los países (periodo 1 y periodo 2):

Figura 1: Dendograma obtenido para el primer periodo para países en América Latina

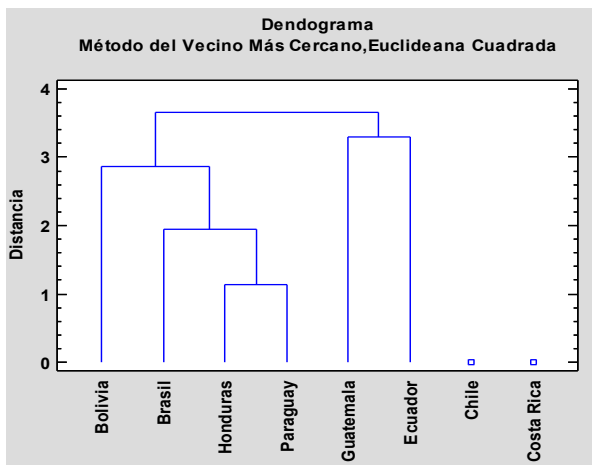

Fuente: Elaboración propia a partir datos CEPALSTAT 2010 
Tabla 4: Grupos resultados tras la aplicación de los variables al Clúster.

\begin{tabular}{|c|c|c|}
\hline Grupo 1 & Grupo 2 & Grupo 3 \\
\hline & & Bolivia \\
Brasil \\
Chile & Costa Rica & $\begin{array}{c}\text { Honduras } \\
\text { Paraguay } \\
\text { Guatemala } \\
\end{array}$ \\
& & Ecuador \\
\hline
\end{tabular}

Fuente: Elaboración propia.

A partir de las variables elegidas para estudiar la pobreza rural, estos son los grupos que se han formado en el primer quinquenio:

- País con muy bajas condiciones de pobreza rural, Chile: A pesar de que la población activa dedicada a la agricultora sea alta, la tercera en el primer quinquenio, la incidencia de la pobreza es muy baja. Sin embargo existe una falta de profesionalización dentro del mundo agrícola, ya que la mayor parte de los ingresos rurales se consigue de manera no laboral. Por lo cual, y a pesar de ser un país con una renta agrícola superior a América Latina, los niveles de desigualdad son muy elevados, superando el 0,5 en el coeficiente de Gini.

- País con bajas condiciones de pobreza rural, Costa Rica: Es el país con menor cantidad de población dedicada al rubro agrícola. También tiene una baja pobreza rural en comparación con el resto de América Latina. Sin embargo, como en el caso chileno, gran parte de los ingresos en los hogares rurales pobres son no laborales, por lo que también existe una baja profesionalización de la agricultura.

- Países con condiciones medias de pobreza rural, (Bolivia, Brasil, Honduras,
Paraguay, Guatemala y Ecuador): Existe un alto porcentaje de población ocupada agrícola superando todas el $55 \%$ de población total activa. La incidencia de la pobreza rural ocupada es alta, llegándose a afirmar que a pesar de estar ocupado dentro del mundo agrícola existe una alta probabilidad de tener una renta por debajo de la línea de la pobreza. Sin embargo, la mayor parte de los ingresos son laborales por lo que se afirma que existe un trabajo laboral profesional, pero con pésimas condiciones laborales.

El desarrollo del mundo rural en América Latina ha sido muy disperso. Los diferentes modelos económicos han dado como resultado un mundo agrícola con altos niveles de distintos tipos de pobreza. Por ello, en este primer periodo se observa como dos países que tienen unos indicadores destacan por su bajo nivel de pobreza, no a nivel mundial, pero sí en comparación con el resto de los países estudiados.

\section{Figura 2: Dendograma obtenido para el segundo periodo para países de América Latina}

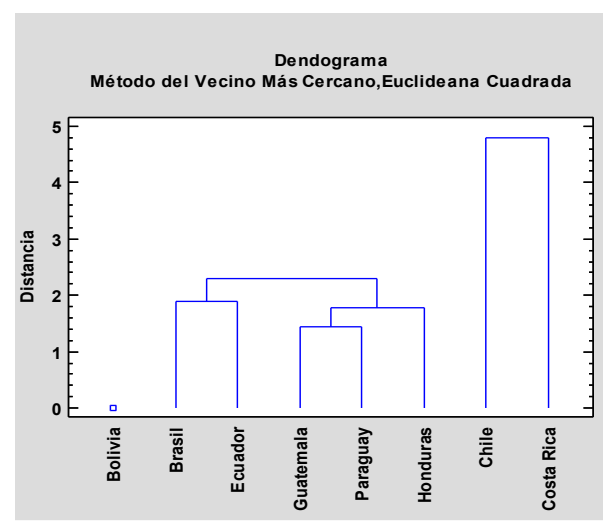

Fuente: Elaboración propia a partir de datos CEPALSTAT 2010. 
Tabla 5: Grupos resultados tras la aplicación de los variables al Clúster.

\begin{tabular}{|c|c|c|}
\hline Grupo 1 & Grupo 2 & Grupo 3 \\
\hline & Brasil & \\
Chile & $\begin{array}{c}\text { Ecuador } \\
\text { Guatemala } \\
\text { Paraguay } \\
\text { Honduras }\end{array}$ & Bolivia \\
& Hondura \\
\hline
\end{tabular}

Fuente: Elaboración propia.

A partir de las variables elegidas para estudiar la pobreza rural, estos son los grupos que se han formado en el segundo quinquenio:

- Países con bajas condiciones de pobreza rural, Chile y Costa Rica: Estos dos países ya fueron destacados dentro del primer quinquenio. La tendencia de ambos países ha sido la reducción de la pobreza rural ocupada y desocupada, mostrando eficacia dentro las políticas planteadas. Sin embargo llaman especialmente la atención dos puntos:

- La población rural pobre en Chile ha aumentado sus ingresos no laborales, haciendo evidente la necesidad de profesionalizar a esta población. Además es el país que más ha bajado su población activa agrícola.

- Costa Rica ha sido el único país de los estudiados que ha aumentado su población activa agrícola en relación al número total de trabajadores activos.

- País con medianas condiciones de pobreza rural (Brasil, Honduras, Paraguay, Guatemala y Ecuador): En todos estos países ha disminuido la población activa agrícola considerablemente con una media de entre 4 y 5 puntos porcentuales. La población rural pobre ocupada ha disminuido considerablemente, como en el caso de Ecuador que ha dis- minuido en 13 puntos porcentuales. Por lo tanto, las políticas de lucha contra la pobreza han sido efectivas, pero no lo suficientes como para mantener a la población agrícola asentada al campo, ya que la pérdida de población ocupada tiende a disminuir.

- Paísconaltascondicionesdepobrezarural, Bolivia: La población activa agrícola ha disminuido considerablemente. Además las condiciones laborales son pésimas, ya que el $73 \%$ de la población ocupada está por debajo de la pobreza. A esta situación, se le suma que es el único país de los estudiados en el que ha aumentado la situación de pobreza en personas desocupadas. Por lo tanto, las políticas sociales rurales en Bolivia no han obtenido los resultados esperados, y en consecuencia ha dado lugar a un éxodo rural.

Las políticas planteadas en este periodo han dado lugar a una continuada reducción de la pobreza, que no ha sido suficiente para mantener a la población activa agrícola. Además los niveles de desigualdad prácticamente no han variado en este tiempo, indicándonos que los modelos de reparto de la tierra y los salarios agrícolas no han variado de forma significativa en este periodo.

\section{DEFINICIÓN DE ECONOMÍA SOCIAL: ESPECIAL REFERENCIA A LOS PRINCI- PIOS FILOSÓFICOS COOPERATIVOS}

Son muchos los países que prevén en su ordenamiento figuras jurídicas sociales o solidarias (Ávila et al., 2008), por lo que resulta difícil dar una definición única de Economía social. Sin embargo, son muchos los autores, (Sykuta y Cook, 2001), (Hart, 1996), (Palacio y Clement, 2007), (Chaves,1999), (Barea, 1990), que 
consideran que las empresas de "Economía social" deben de tener, al menos, cuatro mínimos requisitos:

1. Labor de servir a la sociedad.

2. Autonomía de gestión.

3. Procesos de decisión democrática.

4. Mayor preocupación en los trabajadores que sobre el capital.

Se ha intentado aproximar una definición deEconomía Social(Barea,1990)(Defourny, 2003), (Chávez y Monzon, 2001), (Escobar, 2005), (Coraggio, 2011). No obstante existe una gran complejidad al intentar definirla de manera heterogénea, ya que esta depende del modelo productivo y de las necesidades sociales surgidas por el mismo país. No obstante, existen varias corrientes divididas que engloban a diferentes países con modelos productivos semejantes.

Al crearse varias corrientes, y no existir una definición de la Economía Social global y establecida, tampoco existe un modelo único de tipo figura jurídica determinada. Cada país diseña las suyas y ofrece la consideración oportuna de Economía Social, dependiendo de las necesidades sociales que dicha empresa satisfaga. No obstante, a pesar de estas diferencias culturales e internacionales, sí que existe una corriente sobre la que varios autores, tomando especial atención a Moualert et al. (2007) y Mulgan (2007), determinan que la Economía Social se caracteriza por contribuir al desarrollo territorial por una serie de características en común (López, 2009):

1. Mejor respuesta ante las actividades del entorno.

2. Mayor flexibilidad organizativa y estabilidad operativa.

3. Mayor contribución a la calidad en las prestaciones sociales.

4. Destacada capacidad redistributiva.
5. Capacidad de movilizar recursos.

6. Mayor facilidad de interlocución y cooperación con otros agentes.

Dentro de la Economía Social, el cooperativismo toma especial atención al ser la cooperativa el modelo empresarial organizacional de mayor representación. La Aliance Cooperative International ICA estudia, independientemente del país, estudia el impacto de las cooperativas a nivel internacional. Por lo tanto, y a diferencia de la NR, sí que se puede apreciar una cierta homogeneidad a nivel internacional dentro de los estudios cooperativos. Sin embargo, no existe una leyúnica o política conjunta dela Economía Social ni sobre el cooperativismo, pero sí que se puede hablar de una serie de principios en común que debe de tener una cooperativa para que pueda ser considerada como tal.

El congreso de Manchester viene precedido por una época de estabilidad política entre las democracias occidentales, y con el desmantelamiento del socialismo soviético tras la caída de la URSS. Tras las inquietudes de los movimientos cooperativistas (previamente reunidos en Tokio 1993), se crea una nueva tendencia que ya no están focalizadas en el movimiento obrero y la clásica lucha de clases (Charterina, 1995).

Dicha preocupación ha sido sustituida por la ayuda de personas en peligro de exclusión social y la lucha contra la pobreza (Chaves y Monzón, 2008). Estos ideales son los que se buscan plasmar Manchester 95, identificándose finalmente por unas nuevas normas morales que transformaran el concepto básico de cooperativismo por una nueva organización, más moderna y acorde a sus tiempos. Dichos valores son nombrados como: 
- Igualdad y equidad

- Autoayuda voluntaria y mutua

- Progreso económico y social.

A raíz de esto se abre un discurso en torno a la búsqueda por la honradez, la preocupación por el entorno, el pluralismo e incluso la capacidad de construir. Valores muchos más encaminados a satisfacer las demandas locales a partir de un desarrollo endógeno.

La declaración finalmente se centra en la auto-ayuda; buscando que los trabajadores alcancen los medios de producción; la autoresponsabilidad, centrada a que los socios cooperativistas deben de poner por encima el funcionamiento de la cooperativa si quieren que persista el funcionamiento de la misma, y la interrelación democrática, en busca del bien común y la participación de sus miembros.

Finalmente se concluye con los siete principios cooperativos que servirán como base para desarrollar las figuras jurídicas asociativas:

- Primer Principio: Membresía Abierta y Voluntaria (Puerta abierta).

- Segundo Principio: Control democrático.

- Tercer Principio: Participación económica de los miembros.

- Cuarto Principio: Autonomía e Independencia.

- Quinto Principio: Educación, formación einformación.

- Sexto Principio: Cooperación entreCooperativas.

- Séptimo Principio: Compromiso con la Comunidad.

Diversos autores defienden que estos principios filosóficos dotan a las cooperativas las dotan ciertas ventajas estratégicas, ya que las liga a su entorno (Mozas et al.,
2005) y favorecen tener un mayor conocimiento sobre el mismo, (Vargas, 1995). Estas ventajas competitivas llegan por la implicación de sus socios/empleados, que son autóctonos de la zona. Según Servás 2008, los principios cooperativos también fomentan la innovación ya que reconoce antes los problemas del medio. También Coleman 1988, trata sobre cómo el principio democrático desarrolla un mejor capital humano, ya que cuenta con una mayor interactuación entre sus miembros. Además, la influencia de los principios cooperativos fomenta la financiación local y el valor añadido de los productos locales (Garcia-Gutierrez, 2003).

\section{EL IMPACTO DEL COOPERATIVISMO AGRÍCOLA DENTRO DEL MUNDO RURAL}

Las cooperativas son un tipo de figura jurídica que, independientemente de la legislación autóctona, según el ACI, debe tener unos principios filosóficos comunes. Por lo tanto, sí que existen una serie de características implícitas dentro de la cooperativa independientemente del país en el que se desarrollen.

Los estudios sobre el cooperativismo en Latino América podrían ser más homogéneos, ya que existen instituciones cuya función es el estudio de las cooperativas, como el ACI Américas, o CICOPA. No obstante, es cierto que los diferentes países tienen políticas nacionales sobre como promocionar las cooperativas, lo cual dificulta la cuantificación conjunta de Economía Social en Latino América.

El esfuerzo y la dedicación de los países a destinar fondos y recursos en la Economía Social es muy diversa. En América Latina hay países que ya han destinando departamentos Estatales solo para el desarrollo cooperativo (Tabla 6): 


\section{Tabla 6: Principales organizaciones de Cooperativas en Latino América.}

\begin{tabular}{|l|l|}
\hline $\begin{array}{l}\text { Países con } \\
\text { organizaciones } \\
\text { Cooperativas }\end{array}$ & Nombre de la organización \\
\hline \multirow{2}{*}{ Brasil } & OCB \\
\cline { 2 - 2 } Argentina & UNISOL \\
\hline \multirow{2}{*}{ Colombia } & ASCOOP \\
\cline { 2 - 2 } & CONFECOOP \\
\hline Paraguay & CONPACOOP \\
\hline Uruguay & FCPU \\
\hline Chile & DECOOP \\
\hline Costa Rica & INFOCOOP \\
\hline
\end{tabular}

Fuente: elaboración propia a partir de datos de CICOPA

El cooperativismo, al ser un tipo de organización empresarial enfocada al trabajo colectivo, abarca diferentes rubros productivos, como el sector bancario, el industrial $\mathrm{u}$ organizaciones de consumo. La Organización Internacional del Trabajo, aprovechando el año en que Naciones Unidas declara 2012 como año de las Cooperativas, realiza un estudio con carácter especial "El cooperativismo en América Latina, Una diversidad de contribuciones al desarrollo sostenible". En el mismo se critica que las cifras de la Economía Social son difíciles de recolectar, más aún en América Latina. No obstante, se aproxima un cálculo general de que alrededor de 13.000 cooperativas están afiliadas al Aliance Cooperatives International Américas representado a más de 32 millones de cooperados en toda América Latina.

Los datos ofrecidos por el OIT engloban a los diferentes rubros productivos dentro cooperativismo, enfocando desde un punto de vista general a la cooperativa como un modelo organizacional que lucha contra la pobreza, promociona la inclusión social y mejora las condiciones laborales. Aunque no entren en profundizar en el cooperativismo agrario, sí que se debate sobre el mismo fomentándose como una posibilidad que erradique la pobreza rural, y fomente el desarrollo sostenible medioambiental. Además toma los casos y recomendaciones para los países estudiados (Paraguay, Guatemala, Bolivia, Costa Rica y Honduras).

A parte de este organismo, el ACI Américas realiza afirmaciones generalizados a partir de datos oficiales sobre estudios cooperativos. Sin embargo, no existe un modelo de cuantificación a modo de diagnóstico que permita realizar un estudio comparativo sobre las diferentes realidades de Economía Social dentro de Latino América.

Los estudios actuales sobre Economía Social o Cooperativismo están muy localizados tanto a nivel nacional (Chaves, 2012), como a nivel de casos (Calderón et al, 2012), (Lorenzo, 2015).

En este artículo se toman informes de diferentes organizaciones, tanto nacionales como internacionales, para poder realizar una comparación teórica fundamentada en datos oficiales. Con la dificultad que conlleva conseguir datos oficiales de la Economía Social en América Latina, se toma al menos uno de los países estudiados previamente en la clasificación de pobrezas rurales en América Latina realizadas a partir del método Clúster para comparar cual es el impacto del cooperativismo agrícola dentro del mundo rural. Una vez seleccionados los países, las variables tomadas son las siguientes: 
- Número total de Cooperativas: Total de empresas con modelo Cooperativo (es decir, que siguen los principios filosóficos del Congreso de Manchester 1995) en el país.

- Porcentaje de cooperativas agrarias en relación al total de cooperativas de cada país: En indicador nos muestra la relación que existe entre el cooperativismo y la agricultura mostrando el nivel de especialidad.
- Asalariados empleados por cooperativas: Se toman tanto a socios como trabajadores directos. Indica el total de población activa empleada agrícola cooperada

- Porcentaje de cooperativas agrarias en relación al número total de cooperativas: Este indicador nos muestra la relación entre la agricultura y la población activa cooperada.

Tabla 7: Datos principales de las cooperativas agrícolas en América Latina

\begin{tabular}{|l|r|r|r|r|}
\hline País & Cooperativas & \multicolumn{1}{|c|}{$\begin{array}{c}\text { \% cooperativas } \\
\text { agrarias }\end{array}$} & $\begin{array}{c}\text { Asalariados } \\
\text { Asalariados } \\
\text { totales en } \\
\text { cooperativas } \\
\text { Agrarias } \\
\text { en relación } \\
\text { al total de } \\
\text { empleados en } \\
\text { cooperativa }\end{array}$ \\
\hline Chile (2014) & 952 & $10,50 \%$ & 1748038 & $26,30 \%$ \\
\hline Argentina (2012) & 4977 & $12,50 \%$ & 9362023 & $45,00 \%$ \\
\hline Colombia (2014) & 40888 & $7,20 \%$ & 5.8233477 & $7,90 \%$ \\
\hline Bolivia (2008-10) & 1444 & $18,42 \%$ & 2280015 & $0,69 \%$ \\
\hline Costa Rica (2010) & 530 & 14,7 & 749179 & $34,12 \%$ \\
\hline Honduras (2010) & 2353 & $29,36 \%$ & & - \\
\hline
\end{tabular}

Fuente: Elaboración propia a partir de datos de diversas fuentes ${ }^{4}$

Los modelos cooperativos en Latino América son muy diversos. A pesar, de que la agricultura siempre a estado muy vinculada con la agricultura, no se puede afirmar que haya sido por igual en América latina. El nivel de población activa asalariada en cooperativa varía de forma considerable entre los diversos países estudiados. Mientras que en Bolivia el nivel de vinculación agraria al cooperativismo no alcanza ni el 1 por ciento, en Argentina llega al 45 por ciento, significando que casi uno de cada dos trabajadores cooperados se dedica a la agricultura.

El país que mayor número de cooperados cuenta es Colombia, llegando a casi

4 Estudios oficiales a partir de los que se elabora la tabla: DECOOP (2014). El cooperativismo en Chile.

Ressel, A. B., Silva, N. C., \& Martí, J. P. (2012). El sector de las cooperativas agropecuarias en la República Argentina.

OIT (2012). El cooperativismo en América Latina. Una diversidad de contribuciones al desarrolle s sostenible. CONFECOOP (2014). Informe anual de cooperativas en Colombia. 
Tabla 8: Pobreza por provincias en relación al número de cooperativas en Costa Rica (2009).

\begin{tabular}{|ccccccc|}
\hline Provincia & $\begin{array}{c}\text { Pobreza } \\
\text { extrema }\end{array}$ & Pobreza & $\begin{array}{c}\text { Número de } \\
\text { cooperativas }\end{array}$ & $\begin{array}{c}\text { Población } \\
\text { total }\end{array}$ & $\begin{array}{c}\text { Población } \\
\text { Cooperativa }\end{array}$ & $\begin{array}{c}\text { Tasa de } \\
\text { penetración* }\end{array}$ \\
\hline San José & 3,1 & 12,2 & 143 & 1557371 & 488863 & 31,4 \\
\hline Cartago & - & 16,2 & 33 & 494315 & 6860 & 1,4 \\
\hline Guanacaste & 7,7 & 18,4 & 85 & 279999 & 57711 & 20,6 \\
\hline Puntarenas & 5,6 & 22,6 & 107 & 369180 & 10604 & 2,9 \\
\hline Alajuela & 8,5 & 20,3 & 107 & 841522 & 178810 & 21,2 \\
\hline Limón & 17 & 24,7 & 37 & 420655 & 2176 & 0,5 \\
\hline Heredia & - & 17,8 & 18 & 426186 & 4155 & 1 \\
\hline TOTAL & 4,2 & 21,3 & 530 & 4389228 & 749179 & 17,1 \\
\hline
\end{tabular}

*Índice de penetración cooperativa= (Población activa/población total) X 100

Fuente: OIT 2012

los 6 millones de cooperados según el CONFECOOP. Sin embargo, tampoco existe una especialización cooperada en relación con la agricultura ya que el 7,9 porcentual de la población cooperada trabaja en la agricultura en 2014, mientras que la población activa agrícola era del 65 por ciento.

Costa Rica es el país con mayor número de cooperados $^{5}$ en relación a población activa agrícola. soloel35 por ciento dela población activa trabajaba en lo rural, mientras que la especialización agrícola supera el 34 por ciento. Según el OIT 2012, el Ministerio de Planificación en Costa Rica ya plantea el modelo cooperativo como fórmula para la erradicación de la pobreza. En este país, el Ministerio de Planificación ofrece datos de como la penetración cooperativa favorece la reducción de niveles de pobreza:

5 Personas que trabajan tanto en Cooperativas, como en organizaciones que sigan los principios filosóficos de Manchester 1995
En la tabla se observa como gracias a los modelos cooperativos son las provincias con mayor tasa de penetración cooperativa las que tienen menores niveles de pobreza. De esta forma el ministerio de planificación de Costa Rica apuesta por una política de lucha contra la pobreza con unos objetivos predeterminados de apoyo institucional cooperativo representado por el INFOCOOP. Estos resultados también se han visto traducidos en el mundo agrícola, siendo el único país de los estudiados que ha conseguido subir su porcentaje de población agrícola en relación la población activa total.

En Chile se está apostando por modelos cooperativistas creando en 2014 el Ministerio de Economía, Fomento y Turismo chileno un ministerio específico para promocionar la figura de las Cooperativas. En este sentido se puede destacar la línea de investigación que se está llevando a cabo desde el Estado para la promoción Cooperativa, destacando de los informes llevados 
a cabo por la Subsecretaría de Empresas de Menor Tamaño. De esta manera, y a pesar de los bajos índices de pobreza que muestran en relación al resto de países de Latino América, se forma una política para profesionalizar a la población rural que vive por debajo de la línea de la pobreza, y por mantener cierto atractivo en el mundo rural.

RECOMENDACIONES PARA INTRODUCIR LA ECONOMÍA SOCIAL DENTRO DE LA NUEVA RURALIDAD.

Las condiciones Rurales de América Latina tienen unas peculiaridades que las distancia de las realidades europeas o Norte
Americana. Los principales problemas dentro del mundo latinoamericano son la pobreza, la exclusión social, el envejecimiento de la población o la necesidad de profesionalizar el mundo agrícola.

La Economía social o solidaria se plantea como una solución para satisfacer las necesidades locales. Apuesta por el desarrollo endógeno, la cooperación y sobrepone el desarrollo social por encima del desarrollo económico. Por lo tanto, aparecen un conjunto de objetivos comunes en ambas corrientes que las incentivan a interactuar entre sí (Figura 3):

Figura 3: Modelo de inserción del cooperativismo dentro de la nueva ruralidad
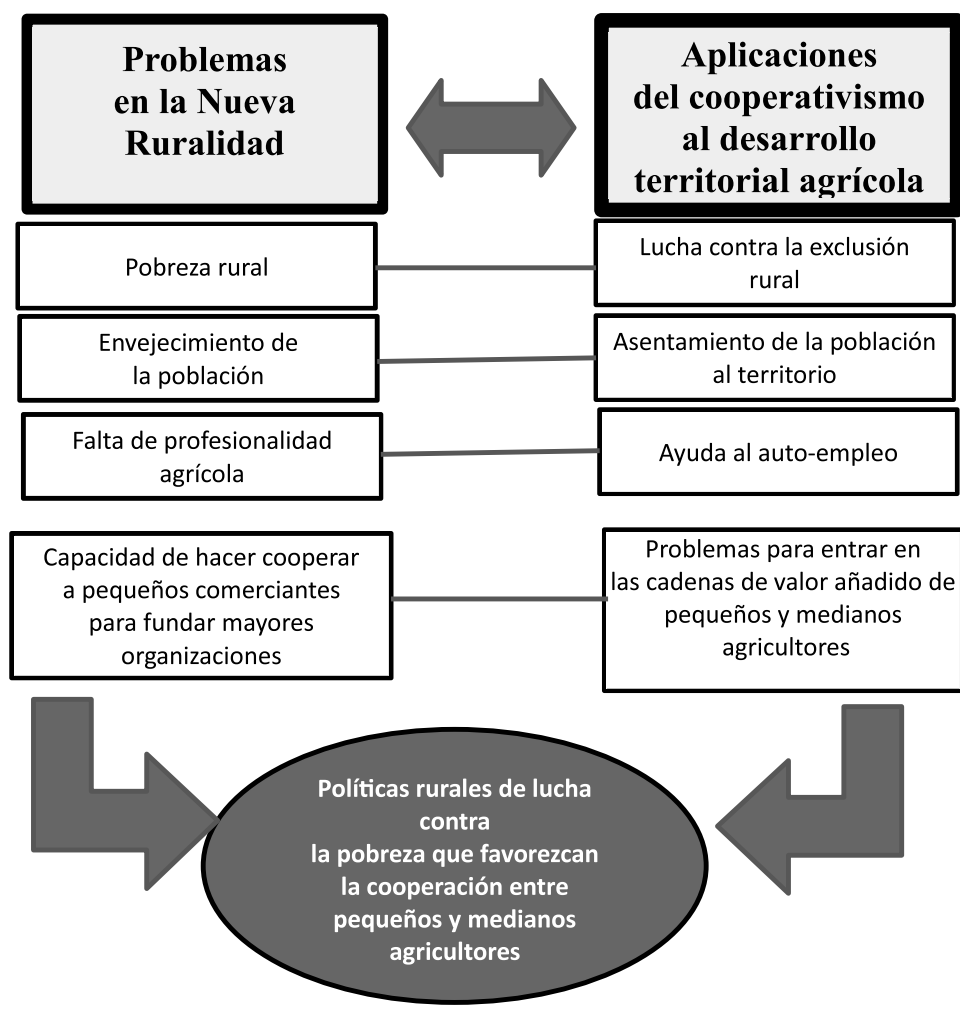

Fuente: Elaboración propia. 
En este artículo se proponen una serie de políticas que podrían resultar interesantes para solventar los problemas de la Nueva Ruralidad, y que a su vez promocione a la Economía Social en general y a las cooperativas en particular:

- Fomentar las instituciones de cooperativas agropecuarias para ayudar a la sinergia de los actores locales públicos y privados.

- Facilitar la información necesaria sobre los beneficios de la cooperación y los métodos de cooperación que están disponibles dentro de las diferentes legislaciones pertinentes.

- Legislar un modelo cooperativo que evite los controles estatales y que incentive profesionalizar el mundo agrícola a través de beneficios fiscales.

- Incentivar la cooperación agrícola entre los pequeños y medianos agricultores tanto para conseguir mayor nego- ciación para la venta ventas y rebajar el coste de los proveedores, como para que puedan aspirar a entrar en las cadenas de valor.

- Formular políticas agrarias con objetivos predefinidos y que tengan un continuo nivel de seguimiento.

- Esclarecer las políticas estructurales agropecuarias por parte de los Organismos Estatales para facilitar la aplicación Leader en los actores locales.

- Crear una red asociativa para los actores estatales que desarrollen una Economía de Escala que permita exportar eficientemente la producción agrícola tanto a nivel nacional como internacional.

- Aumentar la identidad socio-territorial a través de planes de acciones comunes con criterios colaborativos.

- Aumentar la innovación organizativa aplicada a la gestión, producción y generación de valor agregado de la familias agrícolas.

\section{REFERENCIAS BIBLIOGRÁFICAS}

Aráoz, C. (2002). Curso Desarrollo Rural (DRU) 2002 FODEPAL. RLC-FAO. Santiago de Chile.

Ballesteros Babilonia, R. I. (2014). Centro de Estudios para el Desarrollo Rural Sustentable y la Soberanía Alimentaria (CEDRSSA). Estudios e investigaciones: nueva ruralidad; enfoques y propuestas para América Latina. Revista Colombiana de Geografía, 23(1), 189-193.

Barea, J. (1990). Concepto y agentes de la economía social. CIRIEC-España, revista de economía pública, social y cooperativa, (8), 109-117.

Bartlett, W., Cable, J., Estrin, S., Jones, D. C., \& Smith, S. C. (1992). Or-Managed Cooperatives and Private Firms in North Central Italy: An Empirical Comparison. Industrial \& Labor Relations Review, 46(1), 103-118

Benitez, R. O. R. O. (2013). Pobreza rural y políticas públicas en America Latina y el Caribe (No. 364.662 339.46098).

Bernal, E., and Mozas, A. (2005). Las sociedades cooperativas de segundo grado y las TICS. El caso español. Boletín Información Comercial Española, (2.837), 11-24.

Bonnal, P., Bosc, P. M., Díaz, J. M., \& Losch, B. (2003). Multifuncionalidad de la agricultura y nueva ruralidad. Reestructuración de las políticas públicas a la hora de la globalización. Ponencia presentada en el Seminario Internacional El Mundo Rural: Transformaciones y Perspectivas a la luz de la Nueva Ruralidad. Universidad Javeriana. 
Bretón, V. (2005). Los paradigmas de la "nueva" ruralidad a debate: El proyecto de desarrollo de los pueblos indígenas y negros del Ecuador. Revista Europea de Estudios Latinoamericanos Y Del Caribe, 78, 7-30.

Campos-Climent, V., \& Sanchis-Palacio, J. R. (2015). Factores Clave En El Éxito De Las Empresas Agrarias: El Caso De Las Cooperativas Hortofrutícolas En España. Revista Tendencias, 16(2), 174-191.

CEPAL. (2006). América Latina y el Caribe. Observatorio demográfico. [Disponible en] http://www. eclac.org/publicaciones/xml/8/27498/Observatoriodemografico.pdf

CEPAL. (2009). Panorama Social de América Latina. Documento informativo. CEPAL. [Disponible en] http://www.eclac.org/publicaciones/xml/9/37839/PSE2009SintesisLanzamiento.pdf (Consulta: 12-3-12)

CEPAL. (2010). Panorama Social de América Latina. Anexo Estadístico.

CEPAL. (2010). La Hora de la Igualdad. Brechas por cerrar, caminos por abrir. Trigésimo tercer período de sesiones de la CEPAL. Brasilia, 30 de mayo a 1 de junio de 2010. CEPAL. Santiago.

CEPAL. (2010). Estudio Económico para América Latina y el Caribe 2009-2010. Santiago de Chile: Naciones Unidas. Impacto distributivo de las políticas públicas. Naciones Unidas. Santiago. Chile. $339 \mathrm{p}$.

CEPAL. (2011). Panorama Social de América Latina. Documento informativo. CEPAL. [Disponible en] http://www.eclac.org/publicaciones/xml/1/45171/2011-818-PSE-Sintesis-Lanzamiento-WEB. pdf

CEPAL, GTZ. (2009). Taller de expertos "Protección social, pobreza y enfoque de derechos: vínculos y tensiones". América Latina y la protección social: Avances y desafíos para su consolidación (Borrador para comentarios). Octubre de 2009. [Disponible en]: http://www.eclac. cl/dds/noticias/paginas/7/37567/SesionDesafiosPTC.pdf

CEPAL, OIT, FAO. (2010). Políticas de mercado de trabajo y pobreza rural en América Latina. Tomo I. FAO. $324 \mathrm{p}$.

CEPAL, OIT, FAO. (2012). Políticas de mercado de trabajo y pobreza rural en América Latina. Tomo II. FAO. $324 \mathrm{p}$

CICOPA (2014) Informe mundial sobre las cooperativas y el empleo. Quebec.

CONFECOOP (2014). Informe anual de cooperativas.

Defourny, J. (2003). La larga marcha del concepto de economía social. Economía Social, 143-156.

Calderón, B., \& Calderón, J. (2012). Cómo afrontan la crisis las cooperativas en España: comparativa de trayectorias laborales a partir de la Muestra Continua de Vidas Laborales. CIRIEC-España, Revista de Economía Pública, Social Y Cooperativa, 76, 5-26.

Charterina, A. M. (1995). Los valores y los principios cooperativos. REVESCO: revista de estudios cooperativos, (61), 35-46.

Chaves Ávila, R., \& Monzón Campos, J. L. (2008). Panorama de la investigación en economía social. Estudios de Economía Aplicada, 2008, vol. 26, núm. 1, p. 29-56.

Chaves Ávila, R., \& Monzon Campos, J. L. (2012). The social economy in the European Union. 
Chaves Ávila, R. (1999). La economía social como enfoque metodológico, como objeto de estudio y como disciplina científica. CIRIEC-España Revista de economía pública, social y cooperativa, 1999, núm. 33, p. 115-140.

Coraggio, J. L. (2011). Economía social y solidaria. El trabajo antes que el capital, 1, p133.

Climent, V. C., and Ávila, R. C. (2012). El papel de las cooperativas en la Crisis Agraria. Estudio empírico aplicado a la agricultura mediterránea Española. Cuadernos de Desarrollo Rural, 9(69), 175-194.

Coleman, J. S. (1988). Social capital in the creation of human capital. American journal of sociology, S95-S120.

Correa, E. P. (2004). El mundo rural latinoamericano y la nueva ruralidad. Nómadas, (20), 180-193.

De Grammont, H. C. (2004). La nueva ruralidad en América Latina. Revista mexicana de sociología, 279-300.

DECOOP (2014). El cooperativismo en Chile.

Delgado Campos, J. (1999). La nueva ruralidad en México. Investigaciones geográficas, (39), 82-93.

Quijano, M. A. F., \& Correa, E. P. (2003). Mujeres rurales y nueva ruralidad en Colombia. Cuadernos de desarrollo rural, (51).

FAO. (2009). Boom Agrícola y Persistencia de la Pobreza Rural. Estudio de ocho casos.

FAO, CEPAL, OIT. (2010). Políticas del mercado de trabajo y pobreza rural en América Latina. Tomo I. FAORLC. Santiago. Chile. 324 p.

FAO, CEPAL, OIT. (2012). Políticas del mercado de trabajo y pobreza rural en América Latina. II. FAORLC. Santiago. Chile. 353 p.

FAO. (2012). Dinámica del mercado de la tierra en América Latina y el Caribe: Concentración y extranjerización. FAO-RLC. Santiago. Chile. 592 p.

Fontenoy, C. F. (2012). Lo indio, indigenismo y movimiento campesino en el Perú. Revista Andina de Estudios Políticos, 2(1), 1-17.

Gómez, S. (2013). ¿Nueva ruralidad? Un aporte al debate. Estudos Sociedade e Agricultura, Vol, 1.

Graciano da Silva, J., Gomez, E., \& Castañeda, S. (2009). Boom agricola y persistencia de la pobreza rural: Estudio de ocho casos. FAO, Roma (Italia).

Hart, O., \& Moore, J. (1996). The governance of exchanges: members' cooperatives versus outside ownership. Oxford review of economic policy, 12(4), 53-69.

Kay, C. (2009). Estudios rurales en América Latina en el periodo de globalización neoliberal: ¿una nueva ruralidad?. Revista mexicana de sociología, 71(4), 607-645. 
Lattuada, M., \& Nogueira, M. E. (2012). Capacidades estatales y políticas públicas. Una propuesta para el abordaje de las políticas agropecuarias en la Argentina contemporánea (1991-2011). Estudios Rurales, 1(1).

López, J. C. (2009). Sociedades cooperativas y sociedades laborales en España: estudio de su contribución a la creación de empleo y al crecimiento económico. REVESCO: Revista de estudios cooperativos, (98), 35-69.

Lorenzo, F. C., \& Yánez, J. S. N. (2015). La adaptación de las cooperativas agrarias canarias a los cambios económicos acaecidos en el último siglo/Adaptation of agricultural cooperative in Canary Islands to economic changes occurring in the last century. Anuario de Estudios Atlánticos, (61).

Lorenzo, A. M. C., \& Zamora, D. T. (2016). Cooperativismo agrario y turismo rural. Hacia el desarrollo local sostenible. Interações (Campo Grande), 7(11).

Servós, C. M., and Gil, M. I. S. (2008). Los principios cooperativos facilitadores de la innovación: un modelo teórico. REVESCO: Revista de estudios cooperativos, (94), 59-79.

Molotla, F. C. (2013). Cambios ocupacionales en los contextos rurales de México. Revista Facultad de Ciencias Económicas, 21(1), 147-166.

Matás, G. M., Espallardo, M. H., \& Lario, N. A. (2013). La disposición de los socios a invertir en las cooperativas agrarias de comercialización: una aplicación de la teoría de los derechos de propiedad. CIRIEC-Espana, (77), 109.

Moualert, F., Martinelli, F., González, S., \& Swyngedouw, E. (2007). Social Innovation and Governance in European Cities: Urban Development between Path Dependency and Radical Innovation. European Urban and Regional Studies, 14(3), 195-209.

Mozas, A., \& Bernal, E. (2006). Desarrollo territorial y economía social. CIRIEC-España, 55, 125-140.

Mulgan, G., Tucker, S., Ali, R., \& Sanders, B. (2007). Social innovation: what it is, why it matters and how it can be accelerated.

Mejía, A., Castillo, O., \& Vera, R. (2016). Agua potable y saneamiento en la nueva ruralidad de América Latina.

Noa, J. J. L. (2015). El patrimonio cultural indígena como fuente de sentido común desde la perspectiva de la participación comunitaria en Majayara. Panorama, 9(16), 78-90.

OIT (2012). El cooperativismo en América Latina. Una diversidad de contribuciones al desarrollo sostenible.

ODEPA, (2012). Cambio climático Impacto en la Agricultura Helada y Sequía.

Pérez, E. (2001). Hacia una nueva visión de lo ruralidad. ¿Una nueva ruralidad en América Latina? Giarraca, N.(Compiladora) Buenos Aires: CLACSO.

Peña, B. I. M., \& Herrera, L. K. D. (2016). Experiencia de cooperación en el acompañamiento empresarial integral en el territorio como aporte al proceso de reintegración en el posconflicto. Panorama, 10(18), 67-82.

Ramírez-Miranda, C. (2014). Critical reflections on the New Rurality and the rural territorial development approaches in Latin America Visión crítica sobre los enfoques de la Nueva Ruralidad y el desarrollo territorial rural en América Latina. Agronomía Colombiana, 32(1), 122-129. 
Ressel, A. B., Silva, N. C., \& Martí, J. P. (2012). El sector de las cooperativas agropecuarias en la República Argentina.

Rivera, N. R., \& Campos, J. D. (2012). Territorio y nuevas ruralidades: un recorrido teórico sobre las. East, XXXIV, 77-95.

Soto Baquero, F., Klein, E., \& Fernando Soto Baquero, E. K. (2012). Políticas de mercado de trabajo y pobreza rural en América Latina (No. 331 331.1). e-libro, Corp.

Sykuta, M. E., \& Cook, M. L. (2001). A new institutional economics approach to contracts and cooperatives.

Vargas, A. (1995). Las cooperativas de crédito españolas. Una aproximación empírica a algunos aspectos de su gestión. CIRIEC. España: Revista de economía pública, social y cooperativa, (21), 7-20.

Vidal, S. M., Lajara-Camilleri, N., \& Izquierdo, R. J. S. (2013). La formación en las sociedades cooperativas como factor clave de competitividad en un contexto de concentración e internacionalización de los mercados. In Interciencia (Vol. 38, No. 2, pp. 112-120). Interciencia Association.

Zaar, M. H. (2011). Las políticas públicas brasileñas y la agricultura familiar: quince años del Programa Nacional de Fortalecimento da Agricultura Familiar (PRONAF). Scripta Nova. Revista Electrónica de Geografía y Ciencias Sociales, 15(351)

\section{Para citaciones:}

García-García, F. (2017). Debate sobre la inclusión del cooperativismo dentro de las políticas públicas de la Nueva Ruralidad en América Latina. Panorama Económico, 25, 3, pp. 357-380. 